\title{
Questes
}

Revue pluridisciplinaire d'études médiévales

\section{Les sceaux et la représentation du pouvoir. Quelques questions sur les images sigillaires}

David Dominé-Cohn

\section{OpenEdition}

\section{Journals}

Édition électronique

URL : http://journals.openedition.org/questes/1620

DOI : 10.4000/questes. 1620

ISSN : 2109-9472

Éditeur

Les Amis de Questes

Édition imprimée

Date de publication : 15 janvier 2006

Pagination : 13-20

ISSN : 2102-7188

\section{Référence électronique}

David Dominé-Cohn, «Les sceaux et la représentation du pouvoir. Quelques questions sur les images sigillaires », Questes [En ligne], 8 | 2006, mis en ligne le 01 janvier 2014, consulté le 30 avril 2019. URL http://journals.openedition.org/questes/1620 ; DOI : 10.4000/questes.1620

Ce document a été généré automatiquement le 30 avril 2019

(C) Association des amis de «Questes » 


\title{
Les sceaux et la représentation du pouvoir. Quelques questions sur les images sigillaires
}

\author{
David Dominé-Cohn
}

1 Tout le monde connaît le Sceptre d'Ottokar d'Hergé ${ }^{1}$, l'album des aventures de Tintin où ce dernier est amené à rencontrer un sigillographe, le professeur Alambic. Nous avons tous une idée de ce que peut être un sceau, un objet de cire, souvent rond, sur lequel on a imprimé un texte - la légende - et une image. Des livres jusqu'aux films sur le Moyen Âge, nous retrouvons les sceaux sans pour autant lier cet objet à une représentation effective de l'autorité. C'est cependant un objet obscur dont les pouvoirs, sans nous apparaître clairement, se font sentir.

2 Il n'est pas besoin de revenir sur la longue histoire des sceaux, qui, on le sait, commence en Mésopotamie ; il suffit juste de dire que la fonction première des sceaux est de clore les lettres. Cette image est banale, nous la connaissons tous, c'est celle des lettres de cachets. Un gâteau de cire réunit les deux bouts d'une feuille dont le contenu est ainsi caché, sur la cire encore chaude on a imprimé une marque. La première fonction des sceaux, qui est celle qui encore aujourd'hui marque le plus les esprits, est donc cette fonction de clôture. Nous nous souvenons mieux de cette fonction-là que de toutes les autres car elle est associée dans notre imaginaire à la pratique de la lettre de cachet, qui, sur une simple injonction du Roi, pouvait, sous l'Ancien Régime, envoyer n'importe qui sans jugement en prison. Là encore, on le devine, les sceaux ont un rôle fondamental ; c'est le sceau qui rend la lettre opérante et qui transforme un simple texte en ordre contre lequel aucun appel n'est possible.

3 Toutes les lettres scellées ne sont pas closes, et les lettres qui restent visibles aux yeux de tous sont appelées lettres patentes. Ce sont elles qui anoblissent, c'est-à-dire transforment le statut d'une personne; elles aussi qui pardonnent (avec cette forme particulière que sont les lettres de rémission²). Dans le cas des lettres patentes, comme pour celui des lettres closes, l'autorité est en jeu. 
4 Tous les cas que nous pourrons évoquer nous ramèneront à ce constat: le sceau est porteur d'une autorité qui se manifeste dans le caractère performatif des actes scellés. À l'analyse des fonctions du sceau, qui cherchera à mettre en évidence les principes généraux régissant leur fonctionnement, il faudra ajouter un exemple, pour montrer comment la relation entre le sceau et l'autorité, dont il est à la fois une image et un moyen, se fait dans le cadre d'un jeu subtil sur les formes de l'autorité.

5 L'étude des sceaux est restée une science discrète, souvent étouffée entre l'archivistique et l'histoire, hésitant toujours à donner sa mesure, fût-elle nécessairement limitée. Cette réserve de la sigillographie a conduit à réduire souvent ses recherches à de simples questions d'identification qui, bien qu'érudites, ont en fait éloigné les sceaux de la curiosité des historiens. Considérés comme de simples témoins, les sceaux n'étaient que des signatures de cire sur lesquelles régnait l'évidence du nom.

Le sceau est un objet à la fois porteur d'une image et acteur de l'exercice d'une autorité. En tant qu'il constitue une signature en effet $^{3}$, il appelle une reconnaissance, qui doit être comprise dans les deux sens du terme. Il s'agit d'abord d'une reconnaissance issue d'un acte de mémoire: on reconnaît les armoiries ${ }^{4}$, qui sont très souvent dessinées sur le sceau. Le deuxième acte de reconnaissance est juridique: on reconnaît avoir reçu la lettre. Le scellement d'un acte introduit le sceau dans un ensemble de pratiques qui, au cours du temps, se transforment en droit et en normes. De même que dans l'antique Mésopotamie, au temps des sceaux cylindres, le sceau venait garantir que le contenu de l'objet envoyé était conforme à ce qui avait été convenu, le sceau médiéval atteste que le document, l'acte envoyé, a bien toute la force qui le rend exécutoire.

Le sceau est l'objet qui vient permettre la performance, et, au cœur de cette action où l'autorité est en jeu, la reconnaissance joue un rôle fondamental. L'un des moyens de cette reconnaissance est le couple formé par l'image et la légende, qui, tous deux, permettent une double identification. La légende indique le nom du sigillant : elle décline, le plus souvent, une titulature, et parfois les titres qui fondent l'autorité. L'image, quant à elle, n'est pas une simple illustration : elle permet de rappeler une réalité que la légende n'exprime pas et qui participe elle aussi de l'autorité mise en jeu par l'acte. Ainsi, une abbaye ou un évêque placera sur son sceau l'image du saint fondateur de l'institution ${ }^{5}$. De même, on trouve sur les sceaux de seigneurs des représentations de chevaliers, qui viennent rappeler leur fonction première. Parfois, il existe une variation importante de l'iconographie par rapport à ce qu'on peut attendre ${ }^{6}$. Dans le cas des sigillants pourvus d'une chancellerie importante, la variation met en jeu plusieurs types de sceaux, donc plusieurs types d'autorité.

Ce dernier cas nécessite que l'on s'y attarde : il permet au sigillant de faire varier la représentation mise en jeu par son sceau en fonction des circonstances. On peut vérifier au passage le lien étroit qu'entretient le sceau avec le caractère performatif d'un acte, puisqu'un sceau courant suffit pour un acte courant. La pratique de la chancellerie, qui a pour fonction de recevoir et d'expédier les actes d'une autorité, nous permet de mieux souligner le rôle des sceaux dans la production d'une figure - on serait même tenter de dire une forme - de l'autorité. L'apparition et la diffusion, entre les XII et XIII siècles, d'une représentation en majesté sur les grand sceaux ${ }^{7}$ des Empereurs et des souverains (à commencer par les rois de France et d'Angleterre) nous permet d'éclairer l'évolution de la nature de leur pouvoir8.

8 Il est à noter cependant que l'étude de ces dispositifs ne peut concerner qu'une infime partie des sigillants, quoiqu'elle constitue la plus grande part des sceaux que l'on 
connaisse. Les dispositifs d'autorité n'existent à l'état achevés que dans les chancelleries les plus importantes, comme celles du Pape, de l'Empereur, du Roi de France, du Roi d'Angleterre, et quelques autres princes.

9 Il faut souligner enfin que le sceau est un objet singulier et complexe, dont on n'a pas fini de comprendre tous les enjeux. Michel Pastoureau a montré, au cours de ses nombreuses et importantes études sur les sceaux ${ }^{9}$, qu'ils étaient un des lieux les plus importants de la construction d'une sensibilité et d'une mise en scène de soi $^{10}$. Mais il faut aussi noter que cette mise en scène concerne, plus encore que la question de soi, l'autorité, celle du sigillant et, par ricochet, celle de la personne qui reçoit l'acte scellé. Le sceau se trouve au centre d'une relation d'autorité, manifestée par le choix de la figure dont il est porteur. Il faut donc voir dans le sceau une autorité en mouvement, qui s'actualise à chaque nouvelle réception d'un acte scellé.

10 Les remarques que nous venons de faire sur le rôle des sceaux dans une mise en jeu d'un rapport de force et d'autorité ${ }^{11}$ s'appliquent particulièrement à l'étude des pratiques sigillaires de princes. En effet, si par des études nombreuses nous connaissons depuis près de deux cents ans les sceaux et le fonctionnement des chancelleries les plus importantes, c'est-à-dire les chancelleries d'une autorité souveraine, nous ne connaissons pas bien, et en particulier pour la France, celles des pouvoirs qui viennent immédiatement en-dessous des autorités souveraines ${ }^{12}$.

11 Le cas de la chancellerie des ducs de Bretagne apparait comme un des cas les plus problématiques. Pendant le règne de la dynastie des Montfort (1340-1488), arrivée au pouvoir dans le duché à la fin de la Guerre de Succession de Bretagne $(1340-1380)^{13}$, on a utilisé couramment, sous chaque duc, un sceau le représentant de face, assis, la couronne sur la tête et l'épée à la main. Une telle figure, pour l'historiographie, renvoie sans ambiguïté à l'image du roi en position de juge, que l'on trouve sur les sceaux de majesté. On voit que la question de la relation entre l'image sigillaire et l'autorité se pose ici sans détour : quel est alors le statut de l'image ? Si l'image est la représentation de l'autorité, le sceau représentant les ducs de Bretagne en majesté est en contradiction avec la position réelle de ces ducs, qui sont soumis aux Rois de France, en tant que vassaux immédiats. Or, les sceaux ne peuvent pas être porteurs d'ambiguïté, car, comme le rappelle Michel Pastoureau, ils engagent pleinement la responsabilité du sigillant: la représentation qu'ils portent ne doit pas pouvoir être sujette à discussion. Il $\mathrm{y}$ a bel et bien un problème pour les sceaux utilisés à partir du règne de Jean $\mathrm{V}$, duc de Bretagne (1400-1445) jusqu'à la fin de la dynastie - la duchesse Anne de Bretagne, enfant, en posséda un elle aussi.

La résolution de cette question passe par la nécessité d'affiner les termes du problème. Le sceau qu'utilisent les ducs de Bretagne et sur lequel ils se font représenter en majesté n'est pas leur grand sceau. Cette différence, que l'analyse diplomatique des actes de Jean $\mathrm{V}$ éclaire parfaitement, est complétée par la comparaison des tailles des sceaux ${ }^{14}$. On constate en effet que le grand sceau est le plus grand en taille, et ce pour tous les ducs de la dynastie. Cela nous permet de déduire que le sceau de majesté ne peut renvoyer à une majesté semblable à celle du Roi, car il serait alors incompréhensible que ce sceau ne soit pas le plus important. Le grand sceau représente lui aussi le duc, mais dans le cadre d'une figure équestre, ce qui est conforme à l'usage répandu dans toutes les autres chancelleries princières, et plus généralement chez les autres grands seigneurs. La notion de grand sceau a une importance considérable car elle est, contrairement à celle de sceau de majesté, une notion proprement médiévale, qui fait sens pour les hommes du temps. 
Cette distinction manifeste entre les deux sceaux invite à donner une portée juridique plus restreinte à ce sceau de majesté propre aux ducs de Bretagne.

De plus, les sceaux dits de majesté ne concernent dans leur utilisation que des personnes ou des biens soumis à l'autorité du duc de Bretagne ou, ce qui est plus rare, des situations où l'autorité n'est pas réellement mise en jeu. Le grand sceau, qui est lui aussi rarement utilisé pour des correspondances hors du duché, ne pose pas tant de problèmes d'autorité, car il ne présente aucune image dont une personne recevant l'acte pourrait contester l'autorité mise en jeu. Le sceau de majesté des ducs de Bretagne, lui, n'est envoyé qu'à des personnes qui se trouvent sous la domination sans contestation possible des ducs de Bretagne.

14 Le sceau de majesté - qui n'a en fait que peu de rapport avec le grand sceau des souverains français - dessine la carte d'un espace qui tend petit à petit à être celui du duché de Bretagne, mais qui est en réalité celui sur lequel le duc exerce une autorité incontestable, celle de seigneur direct. Le sceau comme figure de l'autorité est donc le moyen d'un jeu entre plusieurs registres d'autorité. L'image du duc assis et détenant une autorité souveraine vient manifester la volonté réitérée des ducs de Bretagne de ne souffrir aucune ingérence de la part d'une autre autorité dans les affaires internes de leur duché; mais, puisque la formulation d'une telle revendication ne peut se faire directement par le moyen d'un grand sceau avec une représentation en majesté qui serait en confrontation directe avec la majesté des Rois, le sceau de majesté vient proposer une figure intermédiaire de l'autorité. Il s'agit donc d'une tentative des ducs pour formuler au sens propre du mot: donner une forme, une apparence - une autorité "souveraine " interne au duché qu'ils détiennent. L'image du sceau devient le lieu d'un jeu de forme et de représentation d'un rapport de force. Par leur grand sceau, les ducs de Bretagne se présentent aux yeux de tous de la même manière que tous les autres grands seigneurs de l'époque, et par leur sceau de majesté, ils se présentent dans leur duché, là où ils exercent pleinement et sans contestation possible leur autorité, comme des souverains.

15 Le sceau se présente bien comme une figure de l'autorité. Il est le support de l'autorité autant que son moyen. Le fait que l'image se trouve sur le sceau fait que celle-ci prend une force performative, qui lui permet de valider et de permettre des actions que les actes commandent. Mais, inversement, le sceau ne saurait être un sceau s'il n'y avait cette image qui autorise la reconnaissance, moment fondateur de la présence de l'autorité.

La double relation qui unit l'image à l'exercice de l'autorité dans le sceau fait de ce dernier un lieu de jeu sur les formes et les formulations, qui permet de proposer une figure plus complexe de leur autorité. Les sigillants se servent des possibilités matérielles qu'offre le sceau et de la relative liberté laissée à la représentation, malgré le caractère très stéréotypé des moyens.

17 En définitive, le sceau nous apparaît comme le vecteur d'une figure fondamentale de l'autorité au Moyen Âge. Il l'est grâce à l'image dont il est le porteur et devient l'autorité elle-même. Il constitue un cas spectaculaire qui illustre la relation particulière des médiévaux au problème de la figuration et à l'autorité de celle-ci. 


\section{NOTES}

1. HERGÉ (Georges Rémy), Tintin et le sceptre d'Ottokar, Bruxelles, 1938, paru d'abord sous le titre Les Nouvelles aventures de Tintin en Syldavie.

2. Pour une analyse de lettres de rémission, voir Claude GAUVARD, "De grace especial ", dans Crimes, Etat et société à la fin du Moyen Âge, Paris, Publication de la Sorbonne, 1991, 2 volumes.

3. On pourra trouver des pistes sur la question de la signature dans Béatrice FRAENKEL, La signature, genèse d'un signe, Paris, Gallimard, 1992.

4. Sur le problème de la reconnaissance des armoiries on peut se reporter d'abord à l'ensemble de l'œuvre de Michel Pastoureau sur le rôle des armoiries comme élément d'indentification d'une personne. On peut lire, pour une analyse plus précise du rôle du blason comme moyen d'une reconnaissance sociale, et en particulier la reconnaissance du rang et de la légitimité de l'autorité, Hans Belting, Pour une anthropologie des images, Paris, Gallimard, 2004, chapitre IV : «Blasons et portraits».

5. Le cas le plus célèbre exemple de cette pratique est celui donné par l'évêque de Rome qui place sur son sceau - la bulle pontificale - l'image de Pierre, premier évêque et apôtre qui vient légitimer ainsi la prétention à la supériorité dans l'Église du titulaire du siège pétrinien.

6. On peut par exemple penser à l'usage d'une iconographie de la majesté chez les ducs de Bretagne, comme c'est le cas pour tout le $\mathrm{xv}^{\mathrm{e}}$ siècle; nous reviendrons sur ce cas dans la seconde partie de cet article pour une analyse plus détaillée.

7. Les grands sceaux sont les sceaux les plus importants de la chancellerie, ce sont ces sceaux sur lesquels la représentation du sigillant est la plus soignée. Leur usage pour sceller un acte nécessite la plus grande formalité.

8. Robert-Henri BAUTIER, "Echanges d'influences dans les chancelleries souveraines au Moyen Âge, d'après les types du sceau de majesté », dans Académie des Inscriptions et Belles-Lettres, compte rendu des séances, Paris, 1969, pp 192-220 ; Martine DALAS, Corpus des sceaux français, t. II, Les sceaux royaux, Paris, Archives Nationales, 1990.

9. On citera, entre autres, le recueil d'articles paru sous le titre Figures et couleurs, études sur la symbolique et la sensibilité médiévales, Paris, Le Léopard d'or, 1986. On peut ajouter Les sceaux, Typologie des sources du Moyen Âge Occidental, $\mathrm{n}^{\circ}$ 36, Turnhout, Brepols, 1981, et « Les sceaux et la fonction sociale des images", dans L'image, fonctions et usages des images dans l'occident médiéval, Actes du 6e International Workshop on Medieval Societies, 17-23 octobre 1992, Paris, Le Léopard d'or, 1995, pp. 275-307 (Cahiers du Léopard d'or n5).

10. La question d'une mise en scène de la personne à travers une image a été étudiée par Hans Belting, op. cit.

11. Sur la notion de rapport de force, on peut se reporter, pour des remarques à l'ouvrage de Carlo GINZBURG, Rapports de force, Paris, Gallimard, Le Seuil EHESS, 2001. En centrant son analyse sur des textes, en particulier dans la remarquable étude consacrée à la donation de Constantin par Lorenzo Valla, C. G. met en évidence que l'interprétation d'un texte ancien, d'une norme ou d'une image, est liée à un contexte historique déterminé par les rapports de force. La compréhension d'une image proposant la représentation d'une situation d'autorité par exemple doit toujours être rapportée à un contexte qui l'autorise et la valide plus qu'il ne la produit.

12. La question du vocabulaire est ici problématique, car l'autorité mise en jeu est multiple. S'entrecroisent différents registres d'autorité (seigneur apanagé, seigneur féodal - c'est le cas des ducs de Bretagne -, prince de l'Empire), engendrant des mélanges qui permettent à certains 
seigneurs de jouer sur plusieurs registres pour produire leurs représentations. On tend pour les derniers siècles du Moyen Âge à utiliser le terme de "prince " pour qualifier ces seigneurs (les ducs de Berry, de Bourbon, de Bourgogne ou de Bretagne) pourvus d'une autorité hors norme (ce qui n'est pas dépourvu d'équivoque).

13. Pour une histoire générale de la période, Joël coRnETTE, Histoire de la Bretagne et des Bretons, Paris, Le Seuil, 2005, 2 volumes.

14. Emile LEFORT DES YLOUSES, «Les ducs de Bretagne et le sceau de majesté », dans Revue française d'Héraldique et de Sigillographie, t. 65, 1995, p. 72.

INDEX

Keywords : autority, power, seal, image, representation, signature, coat of arms

Mots-clés : autorité, pouvoir, sceau, image, représentation, signature, armoirie 